\title{
Minimal detectable change for mobility and patient-reported tools in people with osteoarthritis awaiting arthroplasty
}

Justine M Naylor ${ }^{1,2,3^{*}}$, Andrew Hayen ${ }^{3}$, Edward Davidson ${ }^{4}$, Danella Hackett ${ }^{5}$, lan A Harris ${ }^{1,2,3}$, Gihan Kamalasena ${ }^{1}$ and Rajat Mittal ${ }^{2}$

\begin{abstract}
Background: Thoughtful use of assessment tools to monitor disease requires an understanding of clinimetric properties. These properties are often under-reported and, thus, potentially overlooked in the clinic. This study aimed to determine the minimal detectable change (MDC) and coefficient of variation per cent (CV\%) for tools commonly used to assess the symptomatic and functional severity of knee and hip osteoarthritis.

Methods: We performed a test-retest study on 136 people awaiting knee or hip arthroplasty at one of two hospitals. The $\mathrm{MDC}_{95}$ (the range over which the difference [change] for $95 \%$ of patients is expected to lie) and the coefficient of variation per cent (CV\%) for the visual analogue scale (VAS) for joint pain, the six-minute walk test (6MWT), the timed up-and-go (TUG) test, the Knee Injury and Osteoarthritis Outcome Score (KOOS) and the Hip Disability and Osteoarthritis Outcome Score (HOOS) subscales were calculated.

Results: Knee cohort $(n=75)$ - The $M D C_{95}$ and CV\% values were as follows: VAS $2.8 \mathrm{~cm}, 15 \% ; 6 M W T 79$ m, 8\%; TUG +/-36.7\%, 13\%; KOOS pain 20.2, 19\%; KOOS symptoms 24.1, 22\%; KOOS activities of daily living 20.8, 17\%; KOOS quality of life $26.6,44$. Hip cohort $(n=61)$ - The $M C_{95}$ and $C V \%$ values were as follows: VAS $3.3 \mathrm{~cm}, 17 \%$; 6MWT 81.5 m, 9\%; TUG +/-44.6\%, 16\%; HOOS pain 21.6, 22\%; HOOS symptoms 22.7, 19\%; HOOS activities of daily living 17.7, 17\%; HOOS quality of life $24.4,43 \%$.

Conclusions: Distinguishing real change from error is difficult in people with severe osteoarthritis. The 6MWT demonstrates the smallest measurement error amongst a range of tools commonly used to assess disease severity, thus, has the capacity to detect the smallest real change above measurement error in everyday clinical practice.
\end{abstract}

Keywords: Osteoarthritis, Arthroplasty, Clinimetrics, Patient-reported outcome measures, Timed mobility tests

\section{Background}

Though there is no gold standard for monitoring the progression of knee or hip osteoarthritis (OA), there is value in monitoring the disease $[1,2]$. Knowledge of the trajectory of disease progression provides clinicians and patients with benchmarks against which the effectiveness of everyday self-management strategies [3] or clinicianprovided interventions can be evaluated $[1,2]$. Furthermore, the timing of knee or hip arthroplasty for people

\footnotetext{
* Correspondence: Justine.Naylor@sswahs.nsw.gov.au

'Orthopaedic Department, Liverpool Hospital, Sydney, Australia

${ }^{2}$ School of Public Health and Community Medicine, University of New South Wales, Sydney, Australia

Full list of author information is available at the end of the article
}

with OA may also be informed by capturing significant deterioration in various health domains of those waitlisted for surgery when wait times are protracted. For example, those waitlisted for surgery may be escalated if there is documented evidence of significant decline since first consenting to the procedure.

There are multiple tools available which capture disease severity based on the symptoms and impairments associated with OA. These include tests of physical function and mobility as well as patient-reported surveys. Thoughtful monitoring of the clinical severity of $\mathrm{OA}$ in an individual using these tools requires knowledge of what changes measured by these tools can be considered real. In order to arrive at a decision, the clinician must first be cognisant 
of the minimum change measured by a given tool that is considered to be more than simple measurement error [4]. This minimum change is referred to as the minimal detectable change (MDC) [5-7] or smallest detectable change (SDC) $[4,8,9]$ and is mathematically (linearly) related to the error of the measurement. Put simply, the MDC or the SDC reflects the smallest within-person change in score that can be interpreted as real and statistically significant [8]. In terms of clinimetrics, the MDC is a metric for reproducibility (specifically, a measure of agreement), and is determined by performing repeat measurements on patients over a short period of time [8]. The short time interval renders significant clinical change between assessments unlikely [8], and it also avoids the problem of response shift - a change in the meaning or a recalibration of an outcome - if the tool captures a patient-reported outcome [10].

Despite their obvious value in interpreting real change at the level of the individual, several recent reviews of tools used to assess OA and arthroplasty patients suggest that the measurement error and MDC for the tools are under-reported or underexplored [11-16]. This study, therefore, aimed to evaluate the MDC of tools commonly used to assess the symptomatic and functional severity of knee or hip OA. Specifically, using a test-retest design, we determined the MDC for the visual analogue scale for pain (VAS Pain) $[1,17,18]$, the timed up-and-go (TUG) test [19], the six-minute walk test (6MWT) [20], the Knee Injury and Osteoarthritis Outcome Score (KOOS) [21] and the Hip Disability and Osteoarthritis Outcome Score (HOOS) [22] in individuals with severe OA. As a secondary aim, we also compared the MDCs for the different tools as the magnitude of error may influence the choice of tool used. The tools included were chosen by a multidisciplinary working party overseeing a State-wide program primarily intended to screen, treat and monitor patients with severe knee or hip OA waitlisted for arthroplasty surgery - the Osteoarthritis Chronic Care Program (OACCP) [23].

\section{Methods}

\section{Study design and participants}

A test-retest study involving individuals waitlisted for total knee arthroplasty (TKA) or total hip arthroplasty (THA) at one of two teaching hospitals was undertaken. Individuals meeting the following eligibility criteria were invited to participate: OA in the index joint; waitlisted within the previous two months; and willingness to attend two assessments separated by a 1-week interval. Participants were ineligible if they were unable to comprehend the study protocol either because of an English language limitation or because of documented dementia, or if they intended to change pharmacological or physical management of their OA within the next week. Participants who reported an exacerbation of symptoms or had an acute illness at the first or second assessment were also subsequently deemed ineligible. The study was approved by The Nepean Blue Mountains Lead Human Research Ethics Committee and all participants provided written, informed consent.

\section{Study protocol}

As per current practice, administrative staff contacted all waitlisted individuals via telephone to provide them with an appointment to the chronic care program for assessment of their joint status and overview of their arthritis management. Those meeting the inclusion criteria for the study were invited to be assessed twice for the purposes of the study. Those agreeing to participate were subsequently screened again by a researcher at the first assessment. Co-morbidities, medication lists, and radiology reports were reviewed as per the screening protocol for the OACCP [23]. Eligible participants then completed timed walk tests and several patient-reported measures. Simple yet standardised instructions regarding the completion of the walk tests and patient-reported measures were given as per the program's procedure manual. The participant was instructed to interpret the surveys in the same way when they repeated them the following week and to wear the same footwear. As recommended for test-retest studies [4], the participant was scheduled another appointment one week later, with a maximum time between appointments of 10 days. A physiotherapist at each hospital attached to the waitlist assessment clinic undertook the second assessment, following the same testing procedures. By having a different tester undertake the second assessment, observer independence (from first to second measurement) was ensured $[24,25]$. Time of testing (morning or afternoon), as per usual clinical practice, was not standardised. Recruitment and testing of participants were staggered across the two sites such that both were completed at the first hospital prior to their commencement at the second.

\section{Specific tests \\ VAS for joint pain}

Numerical scales for pain ranging from 0-10 appear to have fairly consistent interpretation across disease states [1]. Significant disability appears to emerge at scores greater than five (moderate degrees of pain) [1]. Participants were asked to mark the pain they felt in their index joint on average over the past week on a $10 \mathrm{~cm}$ scale anchored by 'none' to 'extreme'. On both weeks, this was completed prior to the walk tests.

\section{Timed mobility}

Timed mobility was assessed using the TUG [19] and the 6MWT [20]. Both these tests are recognised performancebased tests for people with OA or who have undergone 
TKA or THA [14,16,26-28]. The TUG was assessed using an armchair (45 cm seat height); the time taken (seconds) to stand from sitting, walk three 3 meters as fast and safely as possible with or without a walking aid, turn, return to the chair and sit down was assessed. A minimum two tests was performed with the fastest time included in the analysis. The 6MWT was conducted on a $30 \mathrm{~m}$ flat track. Participants were instructed to perform each lap 'as fast, but safely as possible' and asked not to stop at each end unless a rest was required [29]. Participants walked alone unless they were deemed unsafe; walking aids were permitted if aids were typically used. The assessor provided standardised verbal encouragement at the end of each 2-lap set. As recommended [20], a practise 6MWT was conducted. This occurred prior to the completion of the surveys. A second test (the test to be included in the analysis as the Week 1 test) was conducted a minimum 30 minutes later, the definitive time dependent upon the individual's symptomatic recovery. Distance covered was recorded in meters.

\section{Joint-specific surveys}

Patient-reported outcomes are commonly used to capture joint-specific pain and function. The KOOS [21] and the HOOS [22] were used here, both derived from the Western Ontario and McMaster Universities Osteoarthritis Index (WOMAC) and developed to capture higher level improvement in younger or more active knee or hip patients. Both surveys have been shown to have face and construct validity and are responsive across a range of conditions $[12,13,21,22,30]$. Whilst the aforementioned VAS for pain was used to capture average joint pain experienced across the week, the KOOS and HOOS capture pain (and impairment) in specific contexts. The surveys include 42 (KOOS) and 40 (HOOS) items covering five patient-relevant joint-related health dimensions referenced to 'the last week': Pain (KOOS 9 items, HOOS 10 items), Other Disease-Specific Symptoms $(7,5)$, Activities of daily living (ADL) Function $(17,17)$, Sport and Recreation Function $(5,4)$, and joint-related Quality of Life (QOL) $(4,4)$. Each item's response is framed within a 5-point Likert scale, ranging from 0 (No Problems) to 4 (Extreme Problems). Each of the five scores is calculated as the sum of the items included. Within each dimension, scores are transformed to a 0-100 scale, with zero representing extreme joint problems and 100 representing no joint problems. In the present study, a priori, the Sport and Recreation Function dimension was excluded based on the knowledge (our own experience and the experience of the developers [21]) that few people wait-listed for arthroplasty engage in higher-level recreational activity, consequently the items (particularly for the KOOS) are generally viewed as less important compared to items in other dimensions [30].
Though not part of the OACCP, the Oxford Knee and Hip Scores (OKS, OHS) [31] were also added to the test protocol for research purposes. For brevity, the reproducibility results of the OKS and OHS are reported elsewhere [32].

\section{Sample size and statistical analyses}

A minimum 50 subjects is a general recommendation for reproducibility studies [33]. Thus, a minimum 50 people with knee OA and 50 with hip OA were planned. As the rate of knee surgery is greater than that for hip surgery at both sites, we anticipated a greater sample for the knee cohort in the timeframe available for recruitment. The presence of systematic bias across the two weeks was investigated using paired t-tests and the relationship between variability (error) and raw score was inspected using Bland and Altman plots [24,25,31]. The MDC for the walk tests and survey scores between the two test days (Week 1 and Week 2) were determined as described below.

For each measure, we calculated the standard error of measurement (SEM) and the 95\% confidence interval $[34,35]$. We also estimated the $90 \%$ confidence interval for a score, which is calculated as $1.645 \times \mathrm{SEM}$; this measure can be used to obtain a $90 \%$ confidence interval for an individual's measurement. Then, for each measure, we calculated the MDC at two levels of confidence, $\mathrm{MDC}_{90}=1.645 \times \sqrt{2} \times \mathrm{SEM}$, and $\mathrm{MDC}_{95}=1.96 \times \sqrt{ } 2 \times \mathrm{SEM}$. The $\mathrm{MDC}_{90}$ and the $\mathrm{MDC}_{95}$ indicate that the difference in two measurements for about $90 \%$ and $95 \%$ of patients respectively will lie in this range. We determined the values at two levels of confidence to aid comparison with the literature. We note that the $\mathrm{MDC}_{95}$ provides the same limits of agreement (LOA) as the Bland and Altman method for assessing agreement [8]. In order to compare agreement indices between tools, we calculated the coefficient of variation per cent (CV\%) using the SEM divided by the Week 1 average [36] multiplied by 100 .

For all outcome variables we checked graphically that the distribution of measurements did not strongly violate the assumption of normality using histograms and Q-Q plots. We found a violation of this assumption for the variable QOL, which was strongly right-skewed. Due to the presence of zero values on the scale, we did not logtransform the QOL data to improve the distribution [25]. For TUG, using the Bland and Altman plots, we found that the variability in measurements was proportional to their level. Therefore, we log-transformed the TUG data [25] and we estimated the $\mathrm{MDC}_{90}$ and $\mathrm{MDC}_{95}$ based on this. In this case, the $\mathrm{MDC}_{90}$ and $\mathrm{MDC}_{95}$ provide an interval in which the ratio of the two measurements for $90 \%$ and $95 \%$ of patients will lie. We also calculated the coefficient of variation for TUG as $\sqrt{\exp \left(S E M^{2}\right)-1}$, where the 
SEM was calculated using the log-transformed data. Finally, we re-estimated the $\mathrm{MDC}_{90}$ and $\mathrm{MDC}_{95}$ for TUG after omitting an outlying observation (for which TUG was $99.9 \mathrm{~s}$ for one hip cohort measurement).

All analyses were conducted using Stata version 13.1, College Station, TX and the study adhered to the guidelines for qualitative research (http://www.biomedcentral. com/authors/rats).

\section{Results}

Over the study period (July - October 2011 Hospital 1, October 2011 - May 2012 Hospital 2), 260 people ( $\mathrm{n}=187$ knee, $n=73$ hip) were waitlisted for surgery. One hundred and ninety-five were eligible to participate; 148 of these consented ( $\mathrm{n}=80$ knee, $\mathrm{n}=68$ hip) and 47 were unable due to work commitments or transport limitations. Sixty-five were ineligible $(\mathrm{n}=17$ non $-\mathrm{OA}, \mathrm{n}=48$ non-English speaking). Of those who provided consent, 136 ( $\mathrm{n}=75$ knee; $\mathrm{n}=61$ hip) attended both assessment sessions; 12 people did not have their second assessment due to illness or transport unavailability. The characteristics of the retained cohort and those for whom repeat data were not available were similar (Table 1). The demographic and health profile of the definitive cohort are summarised in Table 2.

\section{Practise 6MWT and missing data}

One hundred and fifteen participants (68 of 75 knee participants, 47 of 61 hip participants) (85\%) performed a practise $6 \mathrm{MWT}$ on the first testing day prior to undertaking the 'included' Week 1 6MWT; 21 were unwilling to repeat the test on the same day. There were no significant differences between the practise and included 6MWTs for either the knee [346.8 (101.0) vs $350.9 \mathrm{~m}$ (104.0), $\mathrm{p}=0.34$ ] or hip cohort [347.5 m (109.7) vs 343.5 (108.0), $\mathrm{p}=0.28$ ]. Thus, participants who did not complete a practise test remained included in the week-to-week analyses.

Complete week-to-week data sets were not available for all 136 participants. Ten participants $(n=10)$ refused

Table 1 Retained vs Lost cohort

\begin{tabular}{|c|c|c|c|}
\hline & $\begin{array}{l}\text { Retained cohort - } \\
\text { two assessments } \\
\mathrm{N}=136\end{array}$ & $\begin{array}{l}\text { Lost cohort - } \\
\text { one assessment } \\
\mathrm{N}=12\end{array}$ & P-value* \\
\hline Age, yr, mean (sd) & $66.5(9.8)$ & $67.8(8.6)$ & 0.62 \\
\hline Female, n (\%) & $80(59)$ & $9(75)$ & 0.36 \\
\hline $\begin{array}{l}\text { Body Mass Index, } \\
\mathrm{kg} / \mathrm{m}^{2} \text {, mean (sd) }\end{array}$ & $32.5(7.0)$ & $32.7(8.8)$ & 0.94 \\
\hline Timed Up-and-Go, s & $14.0(8.1)$ & $16.7(10.6)$ & 0.61 \\
\hline Six-minute walk test, m & $332.2(112.5)$ & $250.2(84.9)$ & 0.10 \\
\hline $\begin{array}{l}\text { Visual analogue scale, } \\
\text { Pain (index joint), cm }\end{array}$ & $6.7(2.0)$ & $6.4(1.6)$ & 0.46 \\
\hline
\end{tabular}

*Independent sample t-tests or Fisher's Exact Test; sd, standard deviation.
Table 2 Demographic and health profile of the definitive cohort

\begin{tabular}{lll}
\hline & $\begin{array}{l}\text { Knee osteoarthritis } \\
\mathbf{N}=\mathbf{7 5}\end{array}$ & $\begin{array}{l}\text { Hip osteoarthritis } \\
\mathbf{N}=\mathbf{6 1}\end{array}$ \\
\hline Age, years, mean (sd) & $67.6(9.4)$ & $65.2(10.2)$ \\
Female, $\mathrm{n}(\%)$ & $47(63)$ & $33(54)$ \\
Body Mass Index, $\mathrm{kg} / \mathrm{m}^{2}$, & $33.7(7.4)$ & $30.9(6.4)$ \\
mean (sd) & & \\
At least 1 co-morbidity, $\mathrm{n}(\%)$ & $61(83)$ & $54(89)$ \\
Cardiovascular & 50 & 43 \\
Diabetes Mellitus & 20 & 14 \\
Thyroid & 8 & 4 \\
Respiratory & 6 & 9 \\
Central nervous system & 2 & 4 \\
Kidney & 2 & 2 \\
Other & 13 & 16 \\
Other lower limb or & $39(52 \%)$ & $29(48 \%)$ \\
lumbar spine impairment & & $12(20 \%)$ \\
$\begin{array}{l}\text { Previous knee or hip } \\
\text { arthroplasty }\end{array}$ & $17(23 \%)$ & \\
\hline
\end{tabular}

to repeat the 6MWT or the TUG the second week and some did not complete every survey or VAS pain scale at the second assessment due to an administration error. Of those who did complete all surveys, there were no occasions of missing data as all surveys were checked at the time. The minimum sample size analysed for any one tool was 68 and 54 for the knee and hip cohorts respectively.

MDC

The error and agreement indices, including the SEM, the MDC and the CV\% are summarised in Table 3. There were no or minor differences between the means of each test across the weeks (Table 3) and, with the exception of the TUG, the week-to-week differences were not related to the raw scores across the available range. Figures 1 and 2 illustrate the LOA for each tool assessed. The 6MWT demonstrated the lowest error from week-toweek for both the knee and hip cohorts; subsequently, the CV\% (knee, 8\%; hip, 9\%) were the lowest. As the measurement error of TUG was related to level, we report the ratio (week 1 to week 2) in which $90 \%$ and $95 \%$ of measurements should lie. For knees, the $\mathrm{MDC}_{90}$ and $\mathrm{MDC}_{95}$ for TUG were $\pm 30.8 \%$ and $36.7 \%$, meaning that $90 \%$ and $95 \%$ of repeat measurements will be within about $\pm 31 \%$ and $37 \%$ of the original measurement in stable patients respectively. For hips, the $\mathrm{MDC}_{90}$ and $\mathrm{MDC}_{95}$ were $\pm 37.5 \%$ and $44.6 \%$, meaning that $90 \%$ and $95 \%$ of repeat measurements for TUG will be within $\pm 38 \%$ and $45 \%$ of the original measurement in stable patients, respectively. After removing the patient's measurements who had a TUG of 
Table 3 Agreement and reliability indices of objective or patient-reported outcome measures

\begin{tabular}{|c|c|c|c|c|c|c|c|c|}
\hline & $\begin{array}{l}\text { Week } 1 \\
\text { mean (sd) }\end{array}$ & $\begin{array}{l}\text { Week } 2 \\
\text { mean (sd) }\end{array}$ & $\begin{array}{l}\text { Difference } \\
\text { mean (sd) }\end{array}$ & $\begin{array}{l}\text { SEM } \\
(95 \% \mathrm{Cl})\end{array}$ & $\begin{array}{l}90 \% \text { confidence } \\
\text { in score }\end{array}$ & $\mathrm{MDC}_{90}$ & $\mathrm{MDC}_{95}$ & $\mathrm{CV} \%$ \\
\hline \multicolumn{9}{|l|}{ Knee } \\
\hline $6 \mathrm{MWT}(\mathrm{m}) \mathrm{n}=72$ & $344.9(105.6)$ & $348.4(102.3)$ & $-3.5(40.3)$ & 28.5 (24.5 to 34.1$)$ & 46.9 & 66.3 & 79.0 & 8 \\
\hline $\operatorname{TUG}^{*}(\mathrm{~s}), \mathrm{n}=74$ & $12.7(4.7)$ & $13.1(5.4)$ & $-0.4(3.7)$ & NA & NA & $\pm 30.8 \%$ & $\pm 36.7 \%$ & 13 \\
\hline VAS Pain $(\mathrm{cm}), \mathrm{n}=71$ & $6.8(2.0)$ & $6.8(1.9)$ & $0.1(1.6)$ & 1.0 (0.9 to 1.2 ) & 1.7 & 2.4 & 2.8 & 15 \\
\hline \multicolumn{9}{|l|}{ KOOS, $n=68$} \\
\hline Pain & $39.0(18.7)$ & $36.7(18.2)$ & $2.3(10.3)$ & 7.3 (6.2 to 8.8 ) & 12.0 & 17.0 & 20.2 & 19 \\
\hline Symptoms & $38.8(19.2)$ & $39.1(18.8)$ & $-0.4(12.3)$ & 8.7 (7.4 to 10.5$)$ & 40.0 & 20.2 & 24.1 & 22 \\
\hline $\mathrm{ADL}$ & $43.8(18.6)$ & $40.2(19.1)$ & 3.6 (10.6)\# & 7.5 (6.4 to 9.0$)$ & 12.3 & 17.4 & 20.8 & 17 \\
\hline QOL & $21.7(7.7)$ & $23.8(18.8)$ & $-2.7(13.8)$ & 9.6 (8.2 to 11.6$)$ & 15.9 & 22.4 & 26.6 & 44 \\
\hline \multicolumn{9}{|l|}{ Hip } \\
\hline $6 \mathrm{MWT}(\mathrm{m}), \mathrm{n}=54$ & $339.8(107.8)$ & $347.3(105.3)$ & $-7.5(41.6)$ & 29.4 (24.7 to 36.3$)$ & 57.7 & 68.5 & 81.5 & 9 \\
\hline $\operatorname{TUG}^{*}(s), n=56$ & $14.0(7.2)$ & $13.8(6.6)$ & $0.13(3.6)$ & NA & NA & $\pm 37.5 \%$ & $\pm 44.6 \%$ & 16 \\
\hline VAS Pain $(\mathrm{cm}), \mathrm{n}=58$ & $7.1(2.3)$ & $6.8(1.9)$ & $0.4(1.6)$ & $1.2(1.0$ to 1.4$)$ & 1.9 & 2.7 & 3.3 & 17 \\
\hline \multicolumn{9}{|l|}{ HOOS, $n=56$} \\
\hline Pain & $36.0(20.9)$ & $34.4(20.6)$ & $1.6(11.0)$ & 7.8 (6.6 to 9.6) & 12.8 & 18.1 & 21.6 & 22 \\
\hline Symptoms & $42.7(19.4)$ & $38.1(21.8)$ & 4.6 (11.6)\# & 8.2 (6.9 to 10.1$)$ & 13.5 & 19.2 & 22.7 & 19 \\
\hline$A D L$ & 36.7 (20.9) & $34.9(19.7)$ & $1.8(9.0)$ & 6.4 (5.4 to 7.8 ) & 10.5 & 14.8 & 17.7 & 17 \\
\hline QOL & $20.4(20.2)$ & $21.8(21.6)$ & $-1.4(12.5)$ & 8.8 (7.5 to 10.9$)$ & 14.6 & 20.6 & 24.4 & 43 \\
\hline
\end{tabular}

Key: SEM, standard error of measurement with $95 \%$ confidence level; $\mathrm{MDC}_{90}$ and $\mathrm{MDC}_{95}$, minimal detectable change at the $90 \%$ and $95 \%$ confidence level; $\mathrm{CV} \%$, co-efficient of variation per cent; $6 \mathrm{MWT}$, six-minute walk test; TUG, timed up-and-go; VAS, visual analogue scale; KOOS, Knee Injury and Osteoarthritis Outcome Score; HOOS, Hip Disability and Osteoarthritis Outcome Score; ADL, activities of daily living; QOL, quality of life; NA, not applicable (The SEM for TUG is not provided as it is not derived using the same formula due to its skewed distribution); ${ }^{*} T U G$ data were log-transformed - the corresponding MDC $_{90}$ and MDC $_{95}$ are then interpreted as $90 \%$ and $95 \%$ of the measurements being within a ratio of the original measurement. Note, average Week $16 \mathrm{MWT}$ above slightly differs from average Week 1 reported for the comparison between practise and official Week 1 as people who did not have a practise test are included in the average for Week 1 above; \#, significantly different, $\mathrm{p}<0.05$.

99.9 seconds, the $\mathrm{MDC}_{90}$ and $\mathrm{MDC}_{95}$ was $\pm 34.2 \%$ and $40.7 \%$.

\section{Discussion}

Given the complexities and associated burden of quantifying change from one visit to the next [37], one could argue why not simply ask the patient if they have improved or worsened. Though the latter approach may be appealing, in cases where patients may benefit from reporting deterioration - for example, by being escalated to arthroplasty surgery - transparency and quantification of change are required. Further, protracted periods between assessments will undermine both patient and clinician recall, thus, one's ability to recognise change will be in doubt [1]. Consequently, an objective method for interpreting 'change' is required.

The use of the MDC or other indices of error (or reproducibility) to determine thresholds for change is an objective, transparent, simple way to help the clinician monitor change in the individual with OA. Here, we provide the MDC for a range of tools with the $6 \mathrm{MWT}$ demonstrating (in a relative sense) the smallest measurement error across the physical and patient-reported tests assessed. For a change in these tools to be considered 'real', the patient with knee or hip OA respectively would need to demonstrate a minimum change (at the 95\% level of confidence) of at least 79 and $81 \mathrm{~m}$ for the 6 MWT, $3 \mathrm{~cm}$ for VAS Pain, $37 \%$ and $41 \%$ for TUG (from baseline), 20 and 22 for KOOS and HOOS Pain, 24 and 23 for KOOS and HOOS Symptoms, 21 and 18 for KOOS and HOOS ADL, and 27 and 24 for KOOS and HOOS QOL subscales.

The magnitude of the week-to-week variation and MDCs observed here are generally consistent with others. An earlier study observed that $90 \%$ of stable patients with musculoskeletal problems demonstrated a week-to-week change of up to 3 -points (27\%) in their pain rating on an 11-point numeric rating scale [38]. Kennedy et al. [28] reported the $\mathrm{MDC}_{90}$ for the TUG and 6MWT as $2.49 \mathrm{~s}$ and $61.34 \mathrm{~m}$ in their combined TKA and THA cohort. Our $\mathrm{MDC}_{95}$ for the KOOS subscales were generally smaller than the 95\% LOA (equivalent to the $\mathrm{MDC}_{95}$ ) for those reported by Roos and Toksvig-Larsen [21] which ranged from 40 (Symptoms) to 60 (Sport and Recreation subscale), whilst our $\mathrm{MDC}_{95}$ for the HOOS subscales were slightly larger than those reported by Ornetti et al. [39] which ranged from 10 to 20. In terms of how the agreement indices of the KOOS and HOOS Pain, Symptom 


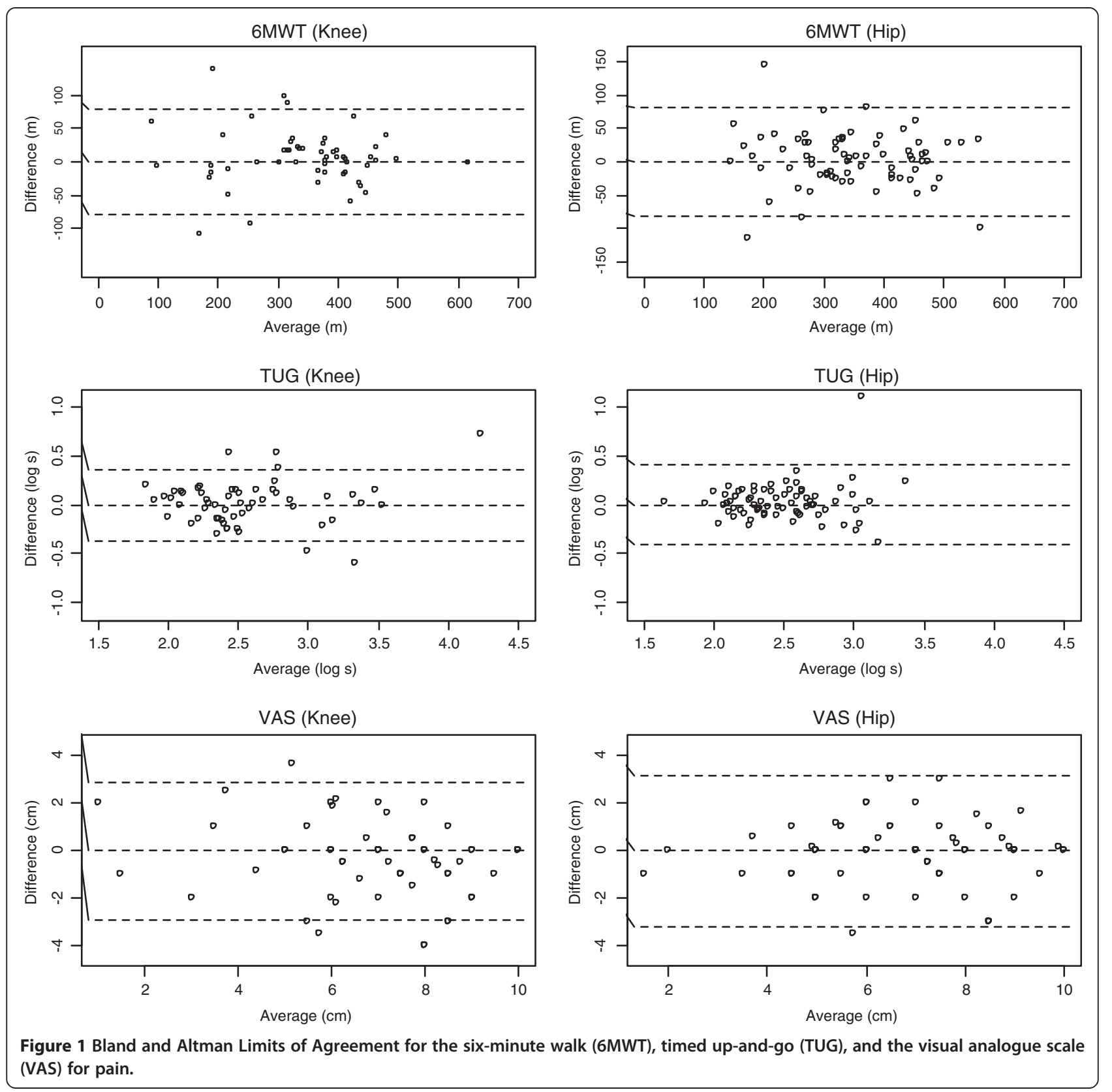

and ADL Function subscales compare with the OKS and OHS, we found that the CV\% were similar (16\% for both OKS and OHS) [32]. These observations are interesting as it appears the greater specificities afforded by the KOOS and HOOS subscales do not guarantee a smaller measurement error compared to a survey that does not differentiate contributions made by pain and functional impairment. It is noteworthy that Impellizzeri et al. [40] reported a much smaller CV (7\%) for the OKS; this is in part explained by the reverse scoring method (12-60, with low scores denoting less pain and impairment) used in their study.
The MDCs are related to the size of the measurement error. Our study design does not allow us to determine whether the error we have observed is due to withinindividual inconsistency in interpretation of survey questions or subclinical, random biological fluctuations in the case of timed walk or continuous pain scale measurements. Nevertheless, the fact remains that 'noise' in outcome measures - whether they be objectively or subjectively measured - will undermine the capacity to monitor disease progression, thus, knowledge of the MDC of each tool is important for reliable interpretation of disease status. Our study design also does not allow us 

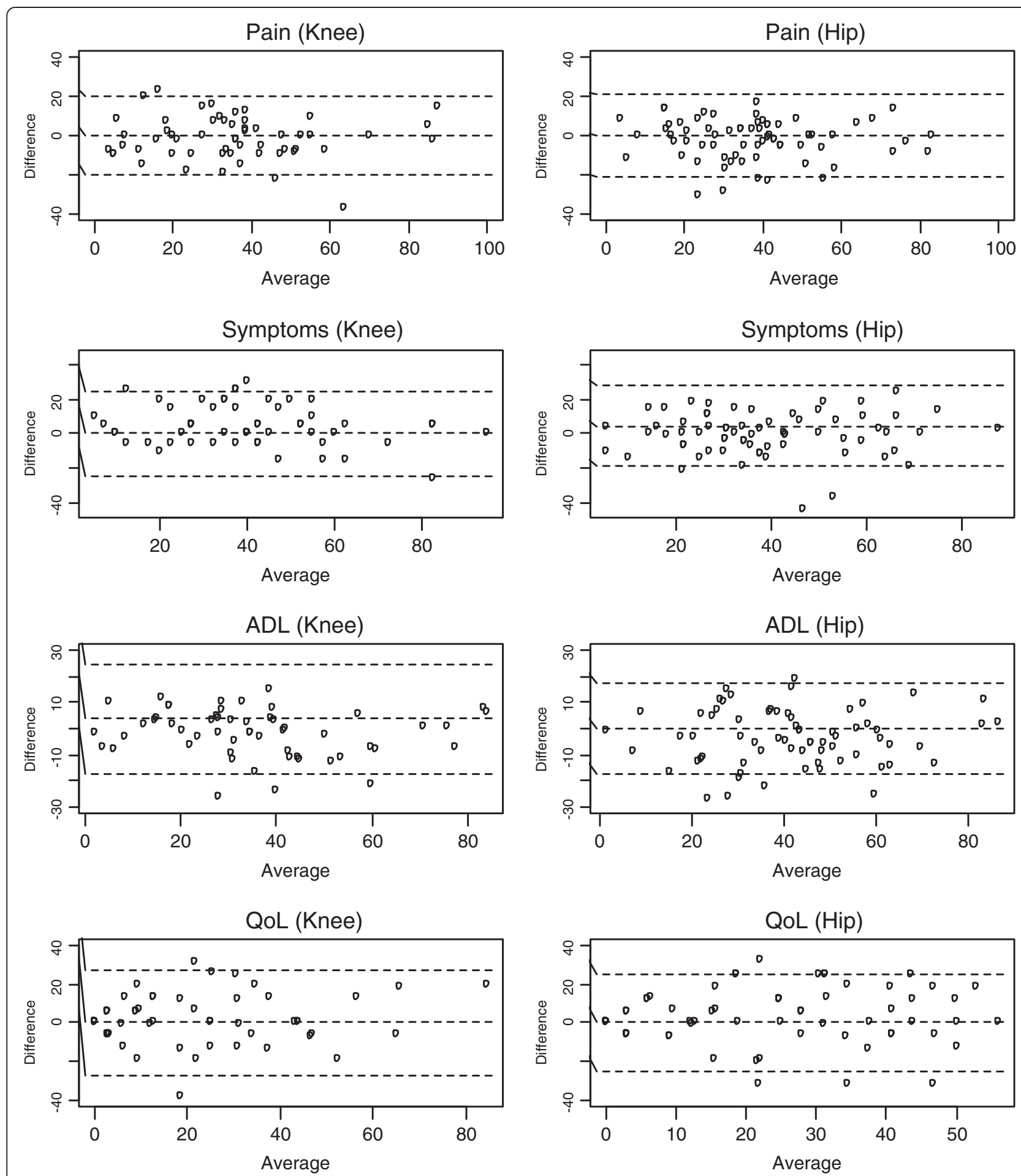

Figure 2 Bland and Altman Limits of Agreement for the Knee Injury and Osteoarthritis Outcome Score (KOOS) and the Hip Disability and Osteoarthritis Outcome Score (HOOS) subscales. Key: ADL - activities of daily living' QoL - quality of life.

to determine what changes are clinically relevant. Whilst the $\mathrm{MDC}_{90}$ and $\mathrm{MDC}_{95}$ values provide clinicians with a threshold about which 'true' change can be considered to have occurred with considerable confidence, these thresholds do not denote thresholds for clinically important changes. Reference to the minimal clinically important difference (MCID) or minimal important change is expected to assist the determination of whether change is 
clinically relevant $[1,8]$. This notwithstanding, it is unclear what these values are for all these tools and their interpretation is contentious given that the MCID appears to vary according to baseline severity and the scale used to determine it, and may be time-dependent [1]. For now, then, the MDCs provide a robust alternative for interpreting change in the clinic.

We acknowledge the strengths and limitations of our study. We examined a well-defined cohort likely to be representative of patients with severe OA waitlisted for arthroplasty. This contention is supported by the observations that: 1 ) the age (68 and 65 yrs), BMI ( 34 and 31), and gender (female, 63 and 54\%) profiles of the knee and hip cohorts respectively, reflect those of the entire patient populations waitlisted for hip or knee arthroplasty at the two sites involved (age, 69 and 65 yrs; BMI, 34 and 30; female gender, 68 and 58\%, knee and hip cohorts respectively) as per the data each site routinely collects for submission to the State's arthroplasty registry (Arthoplasty Clinical Outcome Registry for NSW, ACORN), and; 2) the baseline physical and patient-reported characteristics of our cohorts reflect those reported elsewhere [30-32,39-41]. Our sample size exceeded the minimum recommended sample size for reproducibility studies, we therefore contend the error margins are credible estimates and not unduly influenced by an inadequate sample size. We tested reproducibility under usual care conditions, thus avoiding overly optimistic error estimates. We have provided reproducibility indices of a range of tools commonly used to assess knee and hip $\mathrm{OA}$ in the one study allowing comparisons across the tools. In terms of limitations, we relied on participant perception of their stability in their health status and we did not challenge their declarations that they did not change their medication or physical activity levels between the two test days. Our assumption around stability of health status was necessary as there is no known gold standard for assessing stability in OA [1]. We deliberately avoided the arbitrary use of stability in one of the tools, for example VAS joint pain, as the criterion for participant inclusion in the analysis as this assumes superior reproducibility of the chosen criterion over all others. Nevertheless, 90\% (64/71) of the knee and 84\% (48/61) of the hip cohort demonstrated a test-retest difference of $\leq 2$ points in VAS pain (details not shown in Results). Importantly, these changes align with the weekly changes observed in the LEAP Trial in a cohort of patients with OA who were considered stable [42]. Further support for our contention that the participants were stable is found in the observations (results not shown) that a change in one measure was not reliably associated with a change in another, both in terms of magnitude or direction, suggesting that the changes were, by and large, 'noise'. Regarding unchanged medication and activity levels, participants were aware that their waitlist assessment was to be conducted over two assessments and these would be used by the clinicians to inform their management whilst waiting for surgery. Thus, we contend participants were unlikely to have changed their management knowing that the intention of the assessment (at least from a clinical assessment perspective) was to assess the appropriateness of their current management and to provide a new plan if deemed necessary.

\section{Conclusion}

Knowledge of the MDC values for physical performance and patient-reported tests commonly used to monitor the severity of $\mathrm{OA}$ is necessary for interpreting change within the individual in the context of daily clinical practice. The 6MWD demonstrated the smallest measurement error and, thus, has the capacity to detect the smallest real change above measurement error, making it (potentially) the preferred measurement tool.

\section{Abbreviations \\ 6MWT: Six minute walk test; ADL: Activity of daily living (KOOS, HOOS subscale); CV\%: Coefficient of variation per cent; HOOS: Hip disability and osteoarthritis outcome score; KOOS: Knee injury and osteoarthritis outcome score; LOA: Limits of agreement; MDC 90 : Minimal detectable change $(90 \%$ confidence level - the difference in two measurements for about $90 \%$ of patients will lie in this range).; $\mathrm{MDC}_{95}$ : Minimal detectable change (95\% confidence level - the difference in two measurements for about 95\% of patients will lie in this range); OA: osteoarthritis; OACCP: Osteoarthritis chronic care program; QOL: Quality of life (KOOS, HOOS subscale); SDC: Smallest detectable change; SEM: Standard error of measurement; TKA: Total knee arthroplasty; THA: Total hip arthroplasty; TUG: Timed up-and-go; VAS: Visual analogue scale.}

\section{Competing interests}

The authors declare that they have no competing interests.

\section{Authors' contributions}

JMN conceived and designed the study. JN, GK, RM, DH and ED contributed to data collection. $\mathrm{AH}$ and JN performed the analysis with input from IAH. JN and $\mathrm{AH}$ prepared the manuscript. All authors read, reviewed and approved the manuscript.

\section{Acknowledgements}

We acknowledge the Chair of the OACCP Working Party (Professor David Hunter) and the Project Manager (Mary Fien), the Co-Chair of the Musculoskeletal Network, Matt Jennings, and the Manager, Robin Speerin, all from the Agency for Clinical innovation, for permitting the study to be conducted within the OACCP context. We also acknowledge Loretta Anderson, Felica Lim, Sharon Williams and Lorraine Koenig for their valuable assistance in data collection and recruiting participants.

\section{Author details}

'Orthopaedic Department, Liverpool Hospital, Sydney, Australia. ${ }^{2}$ School of Public Health and Community Medicine, University of New South Wales, Sydney, Australia. ${ }^{3}$ Ingham Institute of Applied Medical Research, Sydney, Australia. ${ }^{4}$ Physiotherapy Department, Nepean Hospital, Sydney, Australia. ${ }^{5}$ Physiotherapy Department, Fairfield Hospital, Sydney, Australia.

Received: 28 March 2014 Accepted: 8 July 2014

Published: 11 July 2014

\section{References}

1. Peat G, Porcheret M, Bedson J, Ward AM: Monitoring in osteoarthritis. In Evidence-Based Medical Monitoring: From Principles to Practice. Edited by Glasziou P, Aronson J, Irwig L. UK: Blackwell Publishing; 2008:335-356. 
2. Allen KD: The value of measuring variability in osteoarthritis pain. J Rheumatol 2007, 34:2132-2133.

3. Allen KD, Oddone EZ, Coffman CJ, Datta SK, Juntilla KA, Lindquist JH, Walker TA, Weinberger M, Bosworth HB: Telephone-based self management of osteoarthritis: a randomized trial. Ann Intern Med 2010, 153:570-579.

4. de Vet HCW, Terwee CB, Knol DL, Bouter LM: When to use agreement versus reliability measures. J Clin Epi 2006, 59:1033-1039.

5. Steffen T, Seney M: Test-retest reliability and minimal detectable change on balance and ambulation tests, the 36-item short-form health survey, and the unified Parkinson disease rating scale in people with parkinsonism. Phys Ther 2008, 88:733-746.

6. Donoghue D, Stokes EK: How much change is true change? The minimum detectable change of the berg balance scale in elderly people. J Rehabil Med 2009, 41:343-346.

7. Stratford PW, Riddle DL: When minimal detectable change exceeds a diagnostic test-based threshold change value for an outcome measure: resolving the conflict. Phys Ther 2012, 92:1338-1347.

8. Terwee CB, Bot SDM, de Boer MR, van der Windt DA, Knol DL, Dekker J, Bouter LM, de Vet HC: Quality criteria were proposed for measurement properties of health status questionnaires. J Clin Epidemiol 2007, 60:34-42.

9. Van Kampen DA, Willems WJ, van Beers LWAH, Castelein RM, Scholtes VAB, Terwee CB: Determination and comparison of the smallest detectable change (SDC) and the minimal important change (MIC) of four-shoulder patient-reported outcome measures (PROMS). J Ortho Surg Res 2013, 8:40.

10. Carr A: Problems in measuring or interpreting change in patient outcomes. Osteoarthritis Cartilage 2002, 10:503-505.

11. Terwee CB, Roorda LD, Knol DL, De Boer MR, De Vet HC: Linking measurement error to minimal important change of patient-reported outcomes. J Clin Epidemiol 2009, 62:1062-1067.

12. Alviar MJ, Olver J, Brand C, Tropea J, Hale P, Pirpiris M, Khan F: Do patient-reported outcome measures in hip and knee arthroplasty rehabilitation have robust measurement attributes? A systematic review. J Rehabil Med 2011, 43:572-583.

13. Collins NJ, Misra D, Felson DT, Crossley KM, Roos EM: Measures of knee function. Arthritis Care Res 2011, 63(S11):S208-S228.

14. Dobson F, Hinman RS, Hall M, Terwee CB, Roos EM, Bennell KL: Measurement properties of performance-based measures to asses physical function in hip and knee osteoarthritis: a systematic review. Osteoarthritis Cartilage 2012, 20:1548-1562.

15. Thorborg K, Roos EM, Bartels EM, Petersen J, Hölmich P: Validity, reliability and responsiveness of patient-reported outcome questionnaires when assessing hip and groin disability: a systematic review. Br J Sports Med 2010, 44:1186-1196.

16. Terwee CB, Mokkink LB, Steultjens MPM, Dekker J: Performance-based methods for measuring the physical function of patients with osteoarthritis of the hip or knee: a systematic review of measurement properties. Rheumatology 2006, 45:890-902.

17. Tubach F, Ravaud P, Baron G, Falissard B, Logeart I, Bellamy N, Bombardier C, Felson D, Hochberg M, van der Heijde D, Dougados M: Evaluation of clinically relevant changes in patient reported outcomes in knee and hip osteoarthritis: the minimal clinically important improvement. Ann Rheum Dis 2005, 64:29-33.

18. Bellamy N, Carette S, Ford PM, Kean WF, le Riche NG, Lussier A, Wells GA, Campbell J: Osteoarthritis antirheumatic drug trials. III. Setting the delta for clinical trials - results of a consensus development (Delphi) exercise. J Rheumatol 1992, 19:451-457.

19. Podsiadlo D, Richardson S: The timed 'Up and Go' Test: a test of basic functional mobility for frail elderly persons. J Amer Geriatric Soc 1991, 39:142-148.

20. Troosters T, Gosselink R, Decramer M: Six minute walking distance in healthy elderly subjects. Eur Respir 」 1999, 14:270-274.

21. Roos EM, Toksvig-Larsen S: Knee injury and Osteoarthritis Outcome Score (KOOS) - validation and comparison to the WOMAC in total knee replacement. Health Qual Life Outcomes 2003, 1:17.

22. Nilsdotter AK, Lohmander S, Klässbo M, Roos EM: Hip disability and osteoarthritis outcome score (HOOS) - validity and responsiveness in total hip replacement. BMC Musculoskelet Disord 2003, 4:10

23. OACCP ACI Musculoskeletal Network. Osteoarthritis Chronic Care Program Model of Care. Agency for Clinical Innovation. 2012, http://www. aci.health.nsw.gov.au/_data/assets/pdf_file/0020/165305/OsteoarthritisChronic-Care-Program-Mode-of-Care.pdf\#zoom=100.
24. Bland JM, Altman DG: Statistical methods for assessing agreement between two methods of clinical measurement. Lancet 1986, 1(8476):307-310

25. Bland JM, Altman DG: Measuring agreement in method comparison studies. Stat Methods Med Res 1999, 8:135-160.

26. Bennell K, Dobson F, Hinman R: Measures of Physical Performance Assessments Self-Paced Walk Test (SPWT), Stair Climb Test (SCT), Six-Minute Walk Test (6MWT), Chair Stand Test (CST), Timed Up \& Go (TUG), Sock Test, Lift and Carry Test (LCT), and Car Task. Arthritis Care Res 2011, 63:S350-S370.

27. Gandhi R, Tsvetkov D, Davey JR, Syed KA, Mahomed NN: Relationship between self-reported and performance-based tests in a hip and knee joint replacement population. Clin Rheumatol 2009, 28:253-257.

28. Kennedy DM, Stratford PW, Wessel J, Gollish JD, Penney D: Assessing stability and change of four performance measures: a longitudinal study evaluating outcome following total hip and knee arthroplasty. BMC Musculoskelet Disord 2005, 6:3.

29. Steffen TM, Hacker TA, Mollinger L: Age- and gender-related test performance in community-dwelling elderly people: six-minute walk test, berg balance scale, timed up \& go test, and gait speeds. Phys Ther 2002, 82:128-137.

30. Roos EM, Lohmander LS: The Knee injury and Osteoarthritis Outcome Score (KOOS): from joint injury to osteoarthritis. Health Qual Life Outcomes 2003, 1:64.

31. Murray DW, Fitzpatrick R, Rogers K, Pandit H, Beard DJ, Carr AJ, Dawson J: The use of the Oxford hip and knee scores. J Bone Joint Surg Br 2007, 89-B:1010-1014.

32. Naylor JM, Kamalasena G, Hayen G, Harris IA, Adie S: Can the oxford scores be used to monitor symptomatic progression of patients awaiting knee or hip arthroplasty? J Arthroplasty 2013, 28:1454-1458.

33. Altman DG: Practical Statistics for Medical Research. London: Chapman \& Hall; 1991.

34. Shrout PE, Fleiss JL: Intraclass correlations: uses in assessing rater reliability. Psychol Bull 1979, 86:420-428.

35. Stratford PW, Goldsmith CH: Use of the standard error as a reliability index of interest: an applied example using elbow flexor strength data. Phys Ther 1997, 77:745-750.

36. Atkinson G, Nevill AM: Statistical methods for assessing measurement error (reliability) in variables relevant to sports medicine. Sports Med 1998, 26(4):217-238.

37. Singh J, Sloan JA, Johanson NA: Challenges with health-related quality of life assessment in arthroplasty patients: problems and solutions. J Am Acad Orthop Surg 2010, 18(2):72-82.

38. Stratford PW, Spadoni G: The reliability, consistency, and clinical application of a numeric pain rating scale. Physiother Can 2001, 53:88-91.

39. Ornetti P, Parratte S, Gossec L, Tavernier C, Argenson JN, Roos EM, Guillemin F, Maillefert JF: Cross-cultural adaptation and validation of the French version of the Hip disability and Osteoarthritis Outcome Score (HOOS) in hip osteoarthritis patients. Osteoarthritis Cartilage 2010, 18:522-529.

40. Impellizzeri FM, Mannion AF, Leunig M, Bizzini M, Naal FD: Comparison of the reliability, responsiveness, and construct validity of 4 different questionnaires for evaluating outcomes after total knee arthroplasty. J Arthroplasty 2011, 26:861-869.

41. Ko V, Naylor JM, Harris IA, Crosbie J, Yeo AET, Mittal R: Is 1-to-1 therapy superior to group- or home-based therapy after knee arthroplasty? A randomized, superiority trial. J Bone Joint Surg Am 2013, 95(21):1942-1949.

42. Hutchings A, Calloway M, Choy E, Hooper M, Hunter DJ, Jordan JM, Zhang Y, Baser O, Long S, Palmer L: The Longitudinal Examination of Arthritis Pain (LEAP) Study: relationships between weekly fluctuations in patient-rated joint pain and other health outcomes. J Rheumatol 2007, 34:2291-2300

doi:10.1186/1471-2474-15-235

Cite this article as: Naylor et al: Minimal detectable change for mobility and patient-reported tools in people with osteoarthritis awaiting arthroplasty. BMC Musculoskeletal Disorders 2014 15:235. 